University of Wollongong

Research Online

Faculty of Engineering and Information

Faculty of Engineering and Information

Sciences - Papers: Part A

Sciences

$1-1-2014$

Intensity ratio approach for 3D profile measurement based on projection of triangular patterns

Zongkai Yang

Huazhong Normal University

$\mathrm{Ke} \mathrm{Wu}$

Huazhong Normal University, University of Wollongong

Jiangtao Xi

University of Wollongong, jiangtao@uow.edu.au

Yanguang Yu

University of Wollongong, yanguang@uow.edu.au

Follow this and additional works at: https://ro.uow.edu.au/eispapers

Part of the Engineering Commons, and the Science and Technology Studies Commons

Research Online is the open access institutional repository for the University of Wollongong. For further information contact the UOW Library: research-pubs@uow.edu.au 


\title{
Intensity ratio approach for 3D profile measurement based on projection of triangular patterns
}

\begin{abstract}
This paper presents an intensity ratio approach for 3D object profilometry measurement based on projection of triangular patterns. Compared to existing intensity ratio approaches, the proposed one is not influenced by the surface reflectivity and ambient light. Moreover, the proposed intensity ratio is point-bypoint-based and thus does not suffer from the influence of surrounding points. The performance of the proposed technique has been tested and the advantages have been demonstrated by experiments. This paper was published in Applied Optics and is made available as an electronic reprint with the permission of OSA. The paper can be found at the following URL on the OSA website: http://www.opticsinfobase.org/ ao/abstract.cfm?uri=ao-53-2-200. Systematic or multiple reproduction or distribution to multiple locations via electronic or other means is prohibited and is subject to penalties under law.
\end{abstract}

\section{Keywords}

patterns, projection, measurement, profile, 3d, approach, ratio, intensity, triangular

\section{Disciplines}

Engineering | Science and Technology Studies

\section{Publication Details}

Z. Yang, K. Wu, J. Xi \& Y. Yu, "Intensity ratio approach for 3D profile measurement based on projection of triangular patterns," Applied Optics, vol. 53, (2) pp. 200-207, 2014. 


\title{
Intensity ratio approach for 3D profile measurement based on projection of triangular patterns
}

\author{
Zongkai Yang, ${ }^{1} \mathrm{Ke} \mathrm{Wu},{ }^{1,2, *}$ Jiangtao $\mathrm{Xi}^{2}{ }^{2}$ and Yanguang $\mathrm{Yu}^{2}$ \\ ${ }^{1}$ National Engineering Research Center for E-Learning, Huazhong Normal University, Wuhan 430079, China \\ ${ }^{2}$ School of Electrical Computer and Telecommunications Engineering, University of Wollongong, \\ Wollongong, NSW 2522, Australia \\ *Corresponding author: kw896@uow.edu.au
}

Received 26 June 2013; revised 29 October 2013; accepted 11 December 2013; posted 16 December 2013 (Doc. ID 192937); published 7 January 2014

\begin{abstract}
This paper presents an intensity ratio approach for 3D object profilometry measurement based on projection of triangular patterns. Compared to existing intensity ratio approaches, the proposed one is not influenced by the surface reflectivity and ambient light. Moreover, the proposed intensity ratio is pointby-point-based and thus does not suffer from the influence of surrounding points. The performance of the proposed technique has been tested and the advantages have been demonstrated by experiments.

(C) 2014 Optical Society of America

OCIS codes: (110.6880) Three-dimensional image acquisition; (150.6910) Three-dimensional sensing; (120.6650) Surface measurements, figure.

http://dx.doi.org/10.1364/AO.53.000200
\end{abstract}

\section{Introduction}

As an effective technology for noncontact 3D shape measurement, digital fringe projection profilometry (DFPP) has attracted extensive research in recent years due to many potential applications. With DFPP, a number of images with special designed patterns are employed to probe the object surface of interest, and the 3D shape of the surface can be extracted from the reflected light patterns.

In order to obtain $3 \mathrm{D}$ object shape information, a number of sinusoidal-pattern based techniques [1-6] have been developed, among which phase-shifting profilometry (PSP) [4] is commonly used due to such advantages as being less sensitive to the surface reflectivity and background illumination. However, PSP usually requires extensive computation, and it also suffers from the influence of nonlinear distortion associated with projectors. Instead of sinusoidal fringe patterns, various other types of image patterns have been proposed and implemented.

$1559-128 \mathrm{X} / 14 / 020200-08 \$ 15.00 / 0$

(C) 2014 Optical Society of America
For example, three images with trapezoidal fringe patterns shifted by one-third of fringe period are employed in $[7,8]$, and a function called intensity ratio is formulate $\bar{b}$ based on the three images. In $[9,10]$ a uniform bright pattern and a linear gray-level pattern are projected, and an intensity ratio map is calculated for each pixel based on these two image patterns. A linearly coded profilometry is presented in $[11,12]$, which uses a saw-tooth-like light pattern to probe the object surface and then utilizes a phaseshifting technique to decode the profile. Jia et al. $[13,14]$ proposed a multiple-step triangular patterns phase-shifting method to profile $3 \mathrm{D}$ object shape. In these methods, triangular patterns are projected, based on which intensity ratio maps are obtained by calculating the normalized intensity difference between different patterns projected. The intensity ratio can be used to extract the height distribution using the triangulation principle. In [15], it is shown that using the intensity ratio, the three-step phase-shifting algorithm can be 3.4 times faster than the traditional three-step method because arctan computation is not required. Unlike the phase calculation in PSP, these approaches obtain the 3D object 
shape by means of the intensity ratios, which are obtained based on the linear operation of the patterns projected. All these intensity-ratio based techniques are advantageous because of reduced computational burden, but fringe patterns are still influenced by nonlinear distortion associated with digital projection, which will then degrade the measurement accuracy. Consequently, intensity ratio-based approaches also need additional techniques to compensate the nonlinear errors [15-17].

As an effort to eliminate the influence of the nonlinear distortion associated with digital projection, a formulation for calculating the $3 \mathrm{D}$ shape is proposed in [18]. The proposed technique uses the same intensity ratio as presented in $[13,14]$, but the $3 \mathrm{D}$ shape calculation is based on the spatial shift estimation, similar to the work presented in [17]. Consequently, the approach in [18] enjoys the advantages of intensity ratio but does not suffer from the nonlinear
$I_{1}(x)$ contains triangular fringes with period $T$ (or pitch) and other patterns are formed by shifting $I_{1}(x)$ along $x$ by an integer multiple of $T / N$, that is, $I_{n}(x)=I_{1}(x-(n T / N))$. A general expression for the intensity ratio using $N$ triangular patterns can be derived as follows [18]:

$$
r_{0}(x)=\frac{\sum_{i=1}^{N}(-1)^{i+1} I_{h, i}(x)-\bmod (N, 2) \times I_{\min }}{I_{v}},
$$

$$
N \geq 2 \text {, }
$$

where $I_{V}=I_{\max }-I_{\min }, I_{\max }$ and $I_{\min }$ are the maximum and minimum intensities for the projected triangular patterns, and $I_{\min }$ can be considered as the background illumination. $I_{h, i}(x)$ is the $i$ th highest intensity values among the $N$ projected patterns at the location of $x$. As $r_{0}(x)$ has the period $T / N$, the following can be used to produce a function that monotonically increases over every fringe period $T[\underline{18}]$ :

$$
r_{s}(x)=\left\{\begin{array}{ll}
(-1)^{k+1} r_{0}(x)+2 \times \operatorname{round}\left(\frac{k-1}{2}\right), & k=1,2, \ldots, 2 N, N \geq 2, N \text { is the odd number } \\
(-1)^{k} r_{0}(x)+2 \times \operatorname{round}\left(\frac{k}{2}\right)-1, & k=1,2, \ldots, 2 N, N \geq 2, N \text { is the even number }
\end{array},\right.
$$

distortion. However, there are still a number of problems associated with the proposed method in [18]. First, these methods usually require the neighborhood pixel properties to calculate the intensity ratio. Second, intensity-ratio based methods are sensitive to either surface reflectivity or background illumination.

In this paper, we propose a new formulation for the intensity ratio, which is also not sensitive to surface reflectivity and background illumination. Besides, the proposed intensity ratio is a point-to-point-based operation and hence is not influenced by the surrounding points. In addition, with the spatial shift estimation method in [18], the 3D object measurement will not be influenced by the system nonlinear distortion.

This paper is organized as follows. In Section 2, the intensity ratio method based on multiple triangular patterns is presented. Section $\underline{3}$ shows experiment results for the proposed method and a comparison with the triangular-pattern spatial shift estimation method. The conclusions are given in Section 4 .

\section{Proposed Method}

\section{A. Existing Intensity Ratio Methods}

The intensity ratio methods proposed in $[14,18]$ are based on the projection of multiple triangular fringe patterns. As the fringe patterns are strips along the $y$ direction, we only consider a cross section along $x$ for simplicity, assuming that $N$ fringe patterns are employed, which are denoted by $I_{n}(x)(n=1,2, \ldots, N)$. where $k$ is referred to as region number, by which each fringe period $T$ is divided into $2 N$ equally spaced segments. $r_{s}(x)$ is denoted as the intensity ratio for reference plane, and the same operations can be carried out to yield $r_{d}(x)$ based on the reflected fringe patterns from the object. In Fig. 1, the intensity ratio calculation based on three-step triangular patterns by employing an existing approach $[14,18]$ is shown as an example. In Fig. 1(a), three-step triangular patterns are projected, and by employing Eqs. (1) and (2), the intensity ratios $r_{0}(x)$ and $r_{s}(x)$ are shown in Figs. 1(b) and 1(c), respectively.

The intensity ratio $r_{0}(x)$ contains $I_{\min }$ and $I_{V}$ in Eq. (1), implying that $r_{0}(x)$ is influenced by the background illumination if an odd number of triangular patterns is employed. However, if even numbers of triangular patterns are projected, $r_{0}(x)$ is not influenced by the background illumination. Whatever odd or even numbers triangular patterns are used, $r_{0}(x)$ is influenced by the peak-to-valley intensity difference $I_{V}$, which will not be a constant in practice due to some factors such as surface reflectivity.

\section{B. Proposed Intensity Ratio}

In order to reduce the influence of background illumination and surface reflectivity on the intensity ratio calculation, we propose a new definition for intensity ratio, which is point-to-point-based, implying that the intensity ratio calculation at each pixel does not suffer from the influence of the surrounding pixels. The proposed intensity ratio is also not sensitive to surface reflectivity and background illumination. 


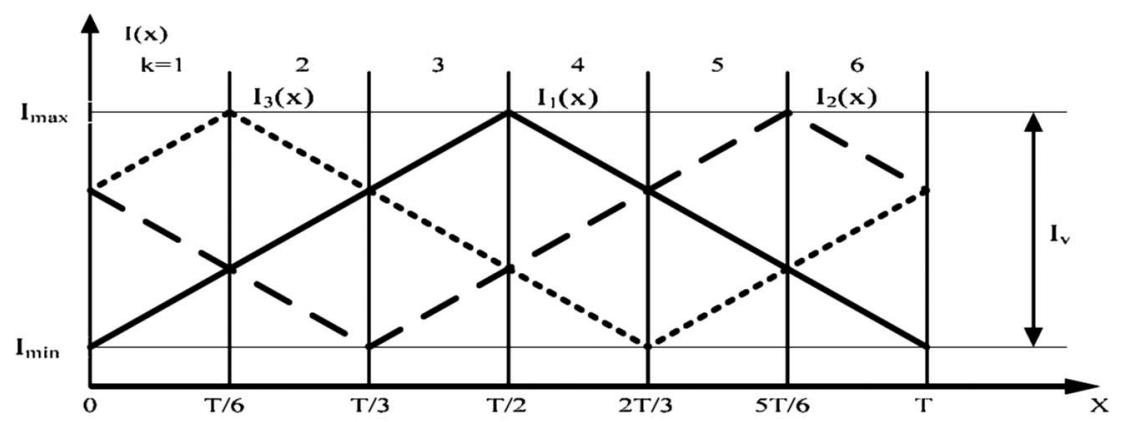

(a)

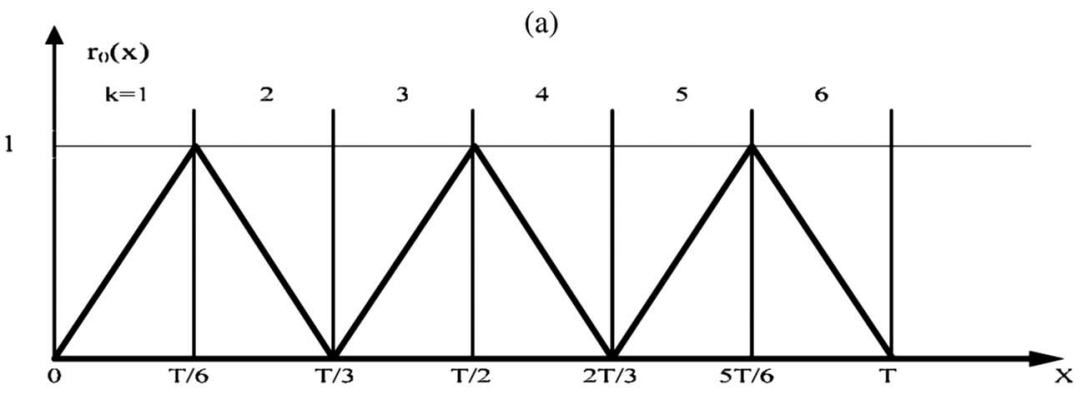

(b)

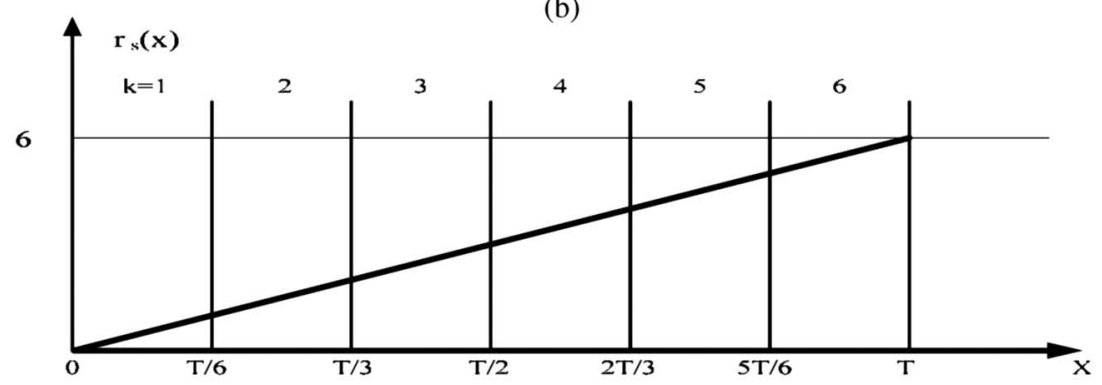

(c)

Fig. 1. Intensity ratio calculation for three-step triangular-pattern method. (a) Cross section of three triangular patterns shifted by one third of the fringe period. (b) Cross section of triangular shape intensity ratio. (c) Cross section of intensity ratio after removal of triangles.

Let us use three-step triangular patterns as an example. The fringe patterns $I_{1}(x), I_{2}(x)$, and $I_{3}(x)$ are still the same as that in Fig. 1(a), which are projected onto the reference plane and object surface, respectively. The three image patterns can be described by the following:

$$
\begin{gathered}
I_{1}(x)= \begin{cases}\frac{2 I_{v}}{T} x+I_{\min }, & x \in\left[0, \frac{T}{2}\right) \\
-\frac{2 I_{v}}{T} x+I_{\min }+2 I_{v}, & x \in\left[\frac{T}{2}, T\right)\end{cases} \\
I_{2}(x)= \begin{cases}-\frac{2 I_{v}}{T} x+I_{\min }+\frac{2 I_{v}}{3}, & x \in\left[0, \frac{T}{3}\right) \\
\frac{2 I_{v}}{T} x+I_{\min }-\frac{2 I_{v}}{3}, & x \in\left[\frac{T}{3}, \frac{5 T}{6}\right) \\
-\frac{2 I_{v}}{T} x+I_{\min }+\frac{8 I_{v}}{3}, & x \in\left[\frac{5 T}{6}, T\right)\end{cases} \\
I_{3}(x)= \begin{cases}\frac{2 I_{v}}{T} x+I_{\min }+\frac{2 I_{v}}{3}, & x \in\left[0, \frac{T}{6}\right) \\
-\frac{2 I_{v}}{T} x+I_{\min }+\frac{4 I_{v}}{3}, & x \in\left[\frac{T}{6}, \frac{2 T}{3}\right) . \\
\frac{2 I_{v}}{T} x+I_{\min }-\frac{4 I_{v}}{3}, & x \in\left[\frac{2 T}{3}, T\right)\end{cases}
\end{gathered}
$$

Note that $r_{0}(x)$ calculated by Eq. ( $\left.\underline{6}\right)$ is still a triangular function with the period $T / 3$, and it has the same
Instead of using Eq. (1), we employ the following to yield $r_{0}(x)$ :

$$
\begin{aligned}
r_{0}(x)= & \frac{I_{h, 1}(x)-I_{h, 2}(x)}{I_{h, 1}(x)-I_{h, 3}(x)} \\
& \begin{array}{ll}
\frac{I_{3}(x)-I_{2}(x)}{I_{3}(x)-I_{1}(x)}=\frac{6}{T} x, & x \in\left[0, \frac{T}{6}\right) \\
\frac{I_{3}(x)-I_{1}(x)}{I_{3}(x)-I_{2}(x)}=-\frac{6}{T} x+2, & x \in\left[\frac{T}{6}, \frac{T}{3}\right) \\
\frac{I_{1}(x)-I_{3}(x)}{I_{1}(x)-I_{2}(x)}=\frac{6}{T} x-2, & x \in\left[\frac{T}{3}, \frac{T}{2}\right) \\
\frac{I_{1}(x)-I_{2}(x)}{I_{1}(x)-I_{3}(x)}=-\frac{6}{T} x+4, & x \in\left[\frac{T}{2}, \frac{2 T}{3}\right) \\
\frac{I_{2}(x)-I_{1}(x)}{I_{2}(x)-I_{3}(x)}=\frac{6}{T} x-4, & x \in\left[\frac{2 T}{3}, \frac{5 T}{6}\right) \\
\frac{I_{2}(x)-I_{3}(x)}{I_{2}(x)-I_{1}(x)}=-\frac{6}{T} x+6, & x \in\left[\frac{5 T}{6}, T\right)
\end{array}
\end{aligned}
$$


shape as that in Fig. 1(b). However, $r_{0}(x)$ does not contain $I_{\max }, I_{\min }$ or $I_{V}$, implying that it is not influenced by the background illumination. The following operation is still used to obtain an intensity ratio function:

$$
\begin{aligned}
r_{s}(x) & =(-1)^{k+1} r_{0}(x)+2 \times \operatorname{round}\left(\frac{k-1}{2}\right), \\
k & =1,2,3,4,5,6 .
\end{aligned}
$$

Obviously $r_{s}(x)$ is also saw-tooth-like, ranging from 0 to 6 , and it also has the same shape as that shown in Fig. 1(c). However, as mentioned above, $r_{s}(x)$ does not contain $I_{\max }, I_{\min }$, or $I_{V}$, and thus is not influenced by the background illumination.

The above can be extended to the case of arbitrary number of fringe patterns. When an even number of triangular patterns is projected, the intensity ratio is given by the following:

$$
r_{0}(x)=\frac{\sum_{i=1}^{N-1}(-1)^{i+1} I_{h, i}(x)}{I_{h, 1}(x)-I_{h, N}(x)},
$$

and

$$
\begin{aligned}
r_{s}(x) & =(-1)^{k+1} r_{0}(x)+2 \times \operatorname{round}\left(\frac{k-1}{2}\right), \\
k & =1,2, \ldots, 2 N, N \geq 3 .
\end{aligned}
$$

When $N$ is an odd number, we have

$$
r_{0}(x)=\frac{\sum_{i=1}^{N}(-1)^{i+1} I_{h, i}(x)}{I_{h, 1}(x)+I_{h, 2}(x)-I_{h, 3}(x)-I_{h, N}(x)},
$$

and

$$
\begin{aligned}
r_{s}(x) & =(-1)^{k} r_{0}(x)+2 \times \operatorname{round}\left(\frac{k}{2}\right)-1, \\
k & =1,2, \ldots, 2 N, N \geq 4 .
\end{aligned}
$$

Also note that $r_{s}(x)$ ranges from 0 to $2 N$ over the full pattern pitch. The same operations can be carried out to yield $r_{d}(x)$ based on the reflected fringe patterns from the object.

From Eqs. (9) and (11), we can see again that the intensity ratio $r_{s}(x)$ does not contain $I_{\max }, I_{\min }$ or $I_{V}$, and hence $r_{s}(x)$ is not influenced by the background illumination. Besides, in Eq. (8), $r_{0}(x)$ is calculated on a point-by-point basis, hence not suffering from the influence of the surrounding points.

\section{Discussion on the Proposed Intensity Ratio}

We have seen that the proposed intensity ratio in Eqs. (9) and (11) is advantageous by immunization from background illumination and the influence of the surrounding points. However, in practice there are many unwanted factors associated with the projection and capture of the image patterns. In this section, we will look into the influence of these factors on the performance of the proposed method.

The actual process in which an image is projected by a digital projector and captured by a digital camera can be described by Fig. 2 [17]. Assuming a fringe pattern $I_{n}(x)$ is fed into the projector, the actual pattern created will be a deformed version, described as follows:

$$
I_{n}^{p}(x)=p\left(I_{n}(x)\right),
$$

where $p(\cdot)$ is a nonlinear function representing the actual response of the projector to the input. Let us utilize $f(x)$ and $b_{1}(x)$ to denote the reflectivity of object surface, and the background illumination, the image pattern reflected from the surface is

$$
I_{n}^{o}(x)=f(x)\left(I_{n}^{p}(x)+b_{1}(x)\right)
$$

The reflected patterns are then captured by the camera with a sensitivity of $\alpha$, which is a constant if we assume the camera response to the light intensity is linear. The image pattern captured by the camera is as follows:

$$
I_{n}^{c}(x)=\alpha\left(I_{n}^{o}(x)+b_{2}(x)\right),
$$

where $b_{2}(x)$ represents the background illumination directly entering the camera. Assuming $\alpha=1$ and combining Eqs. (13) and (14) we have

$$
\begin{aligned}
I_{n}^{c}(x) & =f(x) I_{n}^{p}(x)+f(x) b_{1}(x)+b_{2}(x) \\
& =R(x) I_{n}^{p}(x)+B(x),
\end{aligned}
$$

where $R(x)=f(x)$ and $B(x)=f(x) b_{1}(x)+b_{2}(x)$.

Considering Eq. (15), the intensity ratio $r_{0}(x)$ in Eq. (1) from $\mathrm{N}$-step triangular patterns presented in $[\underline{14}, \underline{18}]$ can be expressed as

$r_{0}(x)=\frac{R(x) \sum_{i=1}^{N}(-1)^{i+1} I_{h, i}^{p}(x)-I_{\min }+B(x)}{I_{v}}$,

$$
N \geq 3 \text {, when } N \text { is even }
$$

and

$$
r_{0}(x)=\frac{R(x) \sum_{i=1}^{N}(-1)^{i+1} I_{h, i}^{p}(x)}{I_{v}},
$$$$
N \geq 4 \text {, when } N \text { is odd }
$$

It is seen that $B(x)$ does not appear in Eq. (17), implying that the even number-step method does not suffer from background illumination. However,

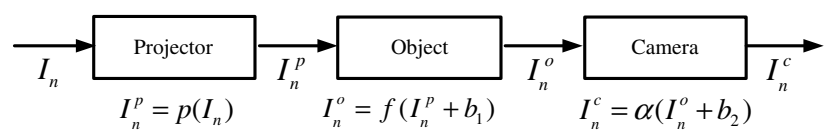

Fig. 2. Camera image generation procedure [17]. 
$R(x)$ appears in both Eqs. (16) and (17), implying that the intensity ratio method in $[14,1 \overline{8}]$ is influenced by the surface reflectivity. Besides, calculation of the intensity ratio requires the peak-to-valley intensity difference $I_{v}$. In practice, $I_{v}$ changes over different fringe period due to the influence of factors like surface reflectivity and noise, thus introducing errors to the intensity ratio.

Now let us consider the intensity ratio method described in Eq. (ㅇ) and Eq. (10). Considering Eq. (15) and for even number-step implementation we have

$$
\begin{aligned}
r_{0}(x) & =\frac{R(x) \sum_{i=1}^{N-1}(-1)^{i+1} I_{h, i}^{p}(x)}{R(x) I_{h, 1}^{p}(x)+B(x)-R(x) I_{h, N}^{p}(x)-B(x)} \\
& =\frac{\sum_{i=1}^{N-1}(-1)^{i+1} I_{h, i}^{p}(x)}{I_{h, 1}^{p}(x)-I_{h, N}^{p}(x)}, \quad N \geq 3 .
\end{aligned}
$$

For odd number-step implementation, we have actual height distribution. The system parameters $H$ and $d$ are calibrated as $1280.0 \mathrm{~mm}$ and $328.9 \mathrm{~mm}$, respectively. The field of vision for CCD camera is $234.5 \mathrm{~mm} \times 175.0 \mathrm{~mm}$. Hence, the equivalent spatial resolution is $0.1684 \mathrm{~mm} /$ pixel. With the intensity ratio proposed in Section 2.B., we use the spatial shift approach to determine the height distribution of the surface as follows:

$$
h(x)=\frac{H \cdot u(x)}{d+u(x)},
$$

where $d$ is the distance between camera and project and $H$ is the distance of the camera to the reference plane. Note that Eq. (20) was originally proposed in [20], where $u(x)$ is the spatial shift between a pixel on the projected image pattern on the reference plane and its corresponding pixels on the object with the same intensity. It has been proved in [18] that Eq. (20) is also valid if $u(x)$ is considere as the

$$
\begin{aligned}
r_{0}(x) & =\frac{R(x) \sum_{i=1}^{N}(-1)^{i+1} I_{h, i}^{p}(x)}{R(x) I_{h, 1}^{p}(x)+B(x)+R(x) I_{h, 2}^{p}(x)+B(x)-R(x) I_{h, 3}^{p}(x)-B(x)-R(x) I_{h, N}^{p}(x)-B(x)} \\
& =\frac{\sum_{i=1}^{N}(-1)^{i+1} I_{h, i}^{p}(x)}{I_{h, 1}^{p}(x)+I_{h, 2}^{p}(x)-I_{h, 3}^{p}(x)-I_{h, N}^{p}(x)}, \quad N \geq 4 .
\end{aligned}
$$

Equations (18) and Eq. (19) do not contain $R(x)$ and $B(x)$, implying that the intensity ratios do not suffer from background illumination as well as surface reflectivity. Moreover, the intensity ratio of a pixel is calculated only based on the intensities of the same pixel on different patterns and hence is not influenced by surrounding ones.

\section{Experiment}

Experiments have been performed to verify the performance of our proposed intensity ratio method. The triangular patterns are generated by a HITACHI CP-X260 projector with a resolution of $1024 \times 768$, and a Duncan Tech MS3100 3CCD camera with a resolution of $1392 \times 1039$ pixels is used to capture the images. The camera and projector are calibrated by means of a reference plane marked by colored grids with their position known a priori. In particular, the camera is calibrated using Bouguet's camera calibration toolbox, which is based on Zhang's calibration method [19]. With the camera calibration toolbox, calibration is achieved by capturing images of a printed checker-board with $15 * 15 \mathrm{~mm}$ grids laced at eight different positions. Similarly, the projector is calibrated using the same eight positions of calibration board as in the camera calibration process. The calibration procedure will yield the intrinsic and extrinsic parameters for both the camera and the projector, with which we can map the pixels in optical devices to real world coordinates to obtain the real spatial shift between a pixel on $r_{s}(x)$ and its corresponding pixels on $r_{d}(x)$.

The first experiment was implemented to determine the accuracy performance associated with the proposed method. First, a flat white board was positioned as the reference plane, yielding $r_{s}(x)$. Then, the same board was moved $20 \mathrm{~mm}$ away from its position as the reference plane, and the reflected pattern is taken as $r_{d}(x)$. The intensity ratio proposed in Eqs. (9) and (11) and Eq. (20) was utilized to calculate the height distribution. The same experiment was repeated using three-, four-, and five-step triangular patterns, each with different pitch values, including 30, 40, and 60 pixels, respectively. For comparison purposes, we also measure the same objects using the intensity ratio proposed in [18]. The measurement results were evaluated by root-meansquare (RMS) error and max error with respect to true height at $700 \times 700$ pixels in the middle of the measured board image. The results of the measurement are shown in Table 1.

In order to compare the proposed method with existing approaches, we also applied the method in [18] to the same object, and the results are shown in Table 1 and Fig. 3. Figure 3 shows the results for the cross section of the board at $y=700$, where the true height is $20 \mathrm{~mm}$ by using the proposed method and the method in [18]. From Table $\underline{1}$ and Fig. 3 , we can see that the proposed intensity ratio method is characterized by smaller measurement 
Table 1. RMS and Max Error (in mm) of Measuring a Flat Board Using the Proposed Approach and Method in [18] with Different Shifting Steps and Pitch Values

\begin{tabular}{lcc}
\hline Pitch (pixels) & 30 & 40 \\
\hline & RMS/Max Error (mm) & 60 \\
\hline The proposed method (3 Steps) & $0.285 / 0.704$ & $0.344 / 0.732$ \\
The method in [18] (3 Steps) & $0.333 / 0.827$ & $0.422 / 1.084$ \\
The proposed method (4 Steps) & $0.267 / 0.665$ & $0.318 / 0.738$ \\
The method in [18] (4 Steps) & $0.281 / 0.673$ & $0.310 / 0.870$ \\
The proposed method (5 Steps) & $0.260 / 0.646$ & $0.262 / 0.592$ \\
The method in [18] (5 Steps) & $0.271 / 0.754$ & $0.280 / 0.686$ \\
\hline
\end{tabular}

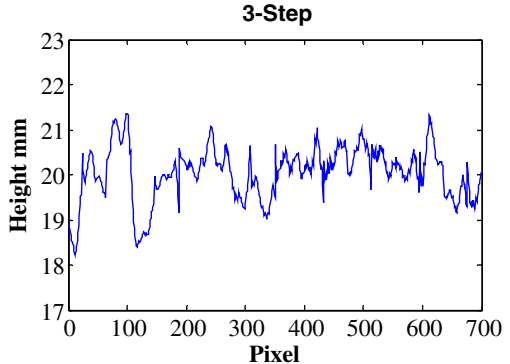

(a)

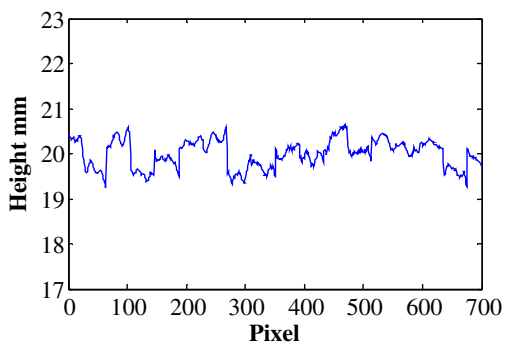

(d)

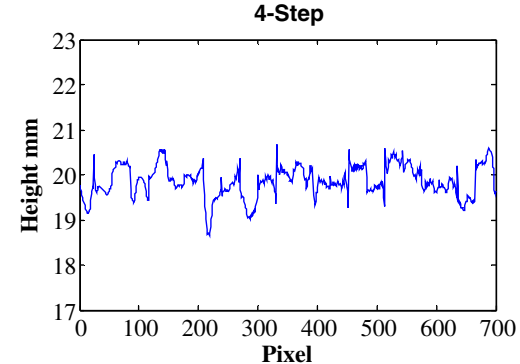

(b)

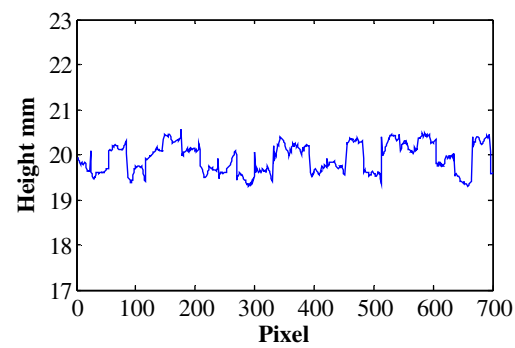

(e)

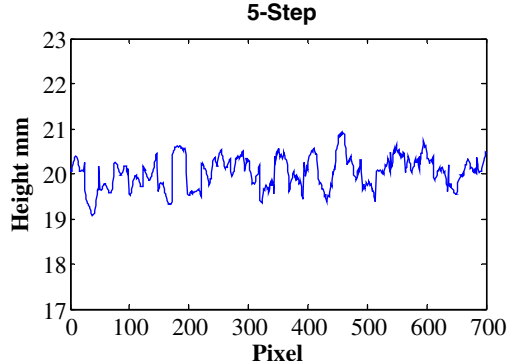

(c)

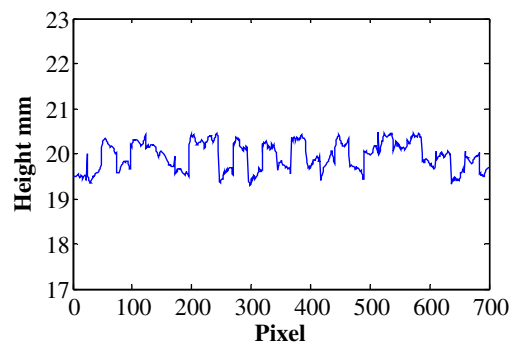

(f)

Fig. 3. Height measurements across the flat board positioned $20 \mathrm{~mm}$ from the reference position using different algorisms with pitch values 60 and different shifting steps. The measurement results are for the three-, four-, and five-step triangular-pattern spatial shifting methods as follows: (a)-(c) Intensity ratio method in [18] for pitch 60, (d)-(f) proposed intensity ratio method for pitch 60.

error than the method proposed in [18] with the same shifting steps and pitches.

The second experiment was conducted to verify the robustness to surface reflectivity of the proposed method. A plate board was used but its surface has different areas, including coated paper, ivory board, blotter paper, and regular A4 print paper, and so the surface reflectivity of the board is different in these areas. Then, the same experiment was repeated as the first experiment does. The results of the measurement are shown in Table 2. From Table 2, we can see that the proposed intensity ratio method is characterized by smaller measurement error than the method in [18] with the same shifting steps and pitches.

In the third experiment, the flat white board was also first positioned as the reference plane. Then, it was moved away from its original position on stepby-step basis with each step of $2 \mathrm{~mm}$ until it is $40 \mathrm{~mm}$ away. The proposed method and the method

Table 2. RMS and Max Error (in mm) of Measuring a Flat Board with Different Surface Reflectivity Using the Proposed Approach and Method in [18] with Different Shifting Steps and Pitch Values

\begin{tabular}{lccc}
\hline Pitch (pixels) & 30 & 40 & 60 \\
\hline & RMS/Max Error (mm) & & \\
\hline The proposed method (3 Steps) & $0.289 / 0.717$ & $0.350 / 0.736$ & $0.378 / 0.766$ \\
The method in [18] (3 Steps) & $0.391 / 0.887$ & $0.475 / 1.144$ & $0.652 / 1.533$ \\
The proposed method (4 Steps) & $0.270 / 0.683$ & $0.322 / 0.706$ & $0.351 / 0.745$ \\
The method in [18] (4 Steps) & $0.321 / 0.793$ & $0.411 / 1.412$ \\
The proposed method (5 Steps) & $0.261 / 0.632$ & $0.287 / 0.899$ & $0.322 / 0.728$ \\
The method in [18] (5 Steps) & $0.290 / 0.762$ & $0.328 / 0.796$ & $0.371 / 0.997$ \\
\hline
\end{tabular}




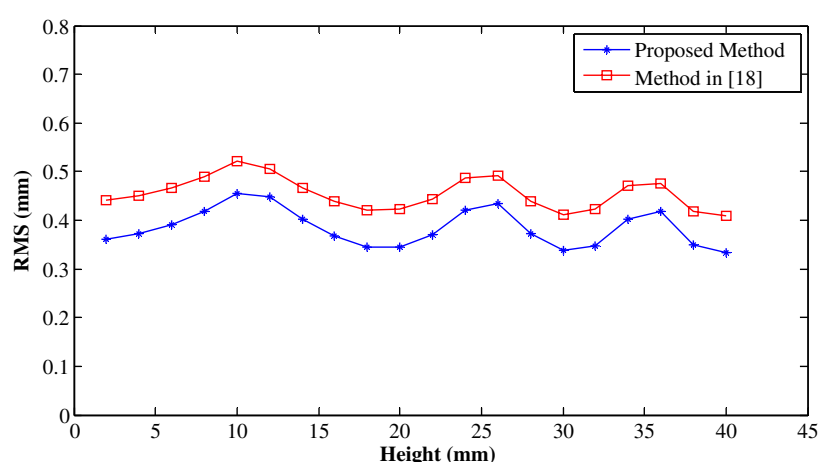

(a)

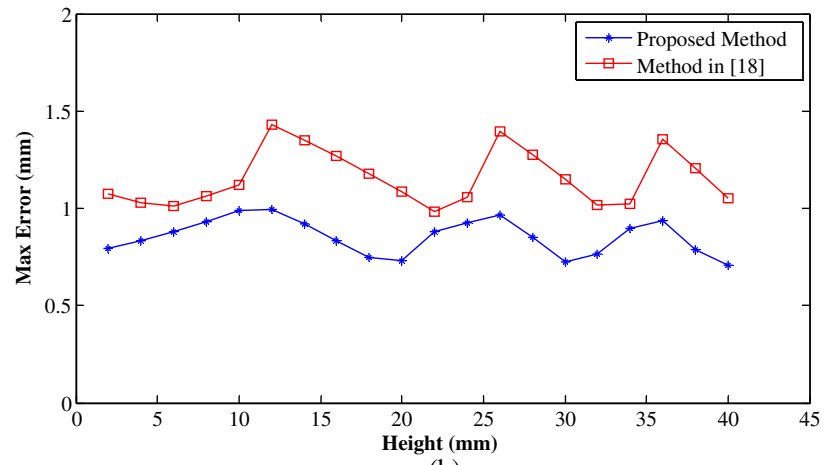

(b)

Fig. 4. Measurement accuracy comparison between proposed three-step intensity ratio method and method in [18] by measuring different height of flat board using a pitch of 40 pixels. (a) RMS comparison between proposed method and method in [18]. (b) Max error comparison between proposed method and method in $[18]$.

in [18] were employed to measure the height of the board for every step of the movement and the measurement accuracy performance is shown in Fig. $\underline{4}$ and

Table 3. RMS and Max Error (in $\mathrm{mm}$ ) of Measuring Different Height of Flat Board Using the Proposed Approach and Method in [18] with Three Steps Triangular Patterns and Pitch 40 Pixels

\begin{tabular}{lcc}
\hline Height $(\mathrm{mm})$ & Proposed Method & Method in [18] \\
\hline 2 & RMS/Max Error $(\mathrm{mm})$ & \\
4 & $0.362 / 0.792$ & $0.441 / 1.076$ \\
6 & $0.373 / 0.833$ & $0.451 / 1.030$ \\
8 & $0.392 / 0.879$ & $0.467 / 1.014$ \\
10 & $0.418 / 0.931$ & $0.490 / 1.066$ \\
12 & $0.454 / 0.989$ & $0.521 / 1.123$ \\
14 & $0.447 / 0.993$ & $0.505 / 1.429$ \\
16 & $0.403 / 0.917$ & $0.468 / 1.352$ \\
18 & $0.367 / 0.835$ & $0.438 / 1.268$ \\
20 & $0.345 / 0.747$ & $0.421 / 1.179$ \\
22 & $0.344 / 0.732$ & $0.422 / 1.084$ \\
24 & $0.369 / 0.877$ & $0.444 / 0.984$ \\
26 & $0.420 / 0.928$ & $0.488 / 1.059$ \\
28 & $0.434 / 0.967$ & $0.491 / 1.393$ \\
30 & $0.372 / 0.849$ & $0.439 / 1.274$ \\
32 & $0.337 / 0.725$ & $0.412 / 1.148$ \\
34 & $0.347 / 0.764$ & $0.423 / 1.018$ \\
36 & $0.403 / 0.895$ & $0.471 / 1.025$ \\
38 & $0.419 / 0.938$ & $0.476 / 1.357$ \\
40 & $0.349 / 0.790$ & $0.418 / 1.208$ \\
\hline & $0.333 / 0.706$ & $0.408 / 1.053$ \\
\hline
\end{tabular}

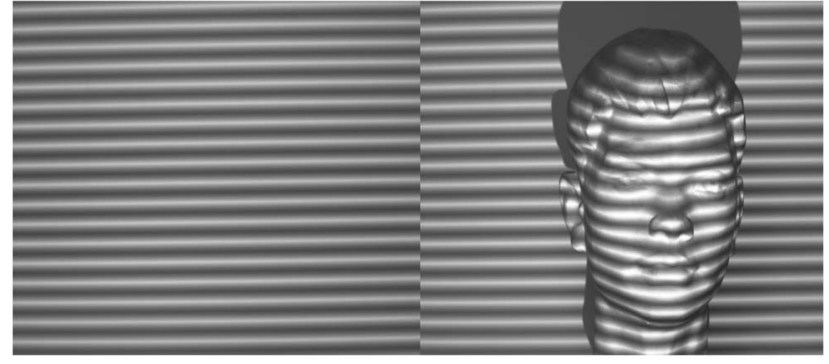

(a)

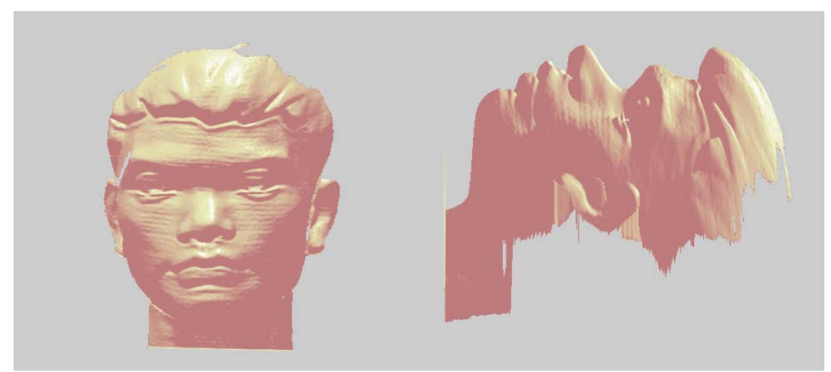

(b)

Fig. 5. 3D reconstruction of a plaster face model by proposed fourstep intensity ratio method with a pitch of 30 pixels. (a) Triangular patterns on the plaster face model and reference plane with pitch 30. (b) Reconstructed 3D models model using surfl() function in MATLAB.

Table 3. The results show again that the proposed method is better than the method in [18] in terms of measurement accuracy.

In the fourth experiment, a plaster face model was used as the target. We used the proposed method with four-step triangular-patterns and with pitch 30 to reconstruct the shape of the model, and the results are depicted in Fig. 5. It is shown that complex objects can be reconstructed by the proposed intensity ratio method.

It should be pointed out that there are a number of issues impacting on the performance of the above proposed method. For example, the experimental setup must be precisely calibrated where the distances among the projector, camera, and reference plane are determined through the calibration process. Besides, the projection being out of focus may also influence the performance, although it may help to yield a better sinusoidal pattern [21]. This is because being out of focus changes fringe shapes, making them nontriangular. The performance of proposed method is also limited by other factors common to the intensity ratio based traditional triangular patterns methods, such as quantization error and intensity noises.

\section{Conclusion}

This paper has presented an intensity ratio formulation for $3 \mathrm{D}$ profile measurement based on the projection of multiple triangular patterns. Compared to the method in the approach [18], the proposed one is not influenced by the surface reflectivity and ambient light. Moreover, the proposed intensity ratio is 
point-by-point-based and thus does not suffer from the influence of surrounding points. Experimental results are also presented, showing that measurement error can be significantly reduced by the proposed method in contrast to the existing approach in $[18]$.

This work was supported by National Natural Science Foundation of China (Nos. 41101406, 61272206, 60903023), Program for New Century Excellent Talents in University (NCET-110654)

\section{References}

1. M. Takeda and K. Mutoh, "Fourier transform profilometry for the automatic measurement of 3D object shapes," Appl. Opt. 22, 3977-3982 (1983).

2. M. Takeda, H. Ina, and S. Kobayashi, "Fourier-transform method of fringe-pattern analysis for computer-based topography and interferometry," J. Opt. Soc. Am. A 72, 156-160 (1982).

3. X. Su and W. Chen, "Fourier transform profilometry: a review," Opt. Laser Eng. 35, 263-284 (2001).

4. V. Srinivasan, H. C. Liu, and M. Halioua, "Automated phasemeasuring profilometry of 3D diffuse objects," Appl. Opt. 23, 3105-3108 (1984).

5. P. S. Huang, C. Zhang, and F. P. Chiang, "High-speed 3D shape measurement based on digital fringe projection," Opt. Eng. 42, 163-168 (2003).

6. C. Guan, L. G. Hassebrook, and D. L. Lau, "Composite structured light pattern for three-dimensional video," Opt. Express 11, 406-417 (2003).

7. S. Zhang and P. S. Huang, "High-resolution, real-time 3D shape acquisition," in IEEE Computer Vision and Pattern Recognition Workshop, (IEEE, 2004), Vol. 3, 28-35.

8. P. S. Huang, S. Zhang, and F.-P. Chiang, "Trapezoidal phaseshifting method for the three-dimensional shape measurement," Opt. Eng. 44, 123601 (2005).
9. B. Carrihill and R. Hummel, "Experiments with the intensity ratio depth sensor," Comput. Vis. Graph. Image Process. 32, 337-358 (1985)

10. T. Miyasaka and K. Araki, "Development of real time 3D measurement system using intensity ratio method," in Proc. ISPRS Commission III, Vol. 34, Part 3B (Photogrammetric Computer Vision, 2002), pp. 181-185.

11. Q. Fang, "Linearly coded profilometry with a coding light that has isosceles triangle teeth: even-number-sample decoding method," Appl. Opt. 36, 1615-1620 (1997).

12. Q. Fang and S. Zheng, "Linearly coded profilometry," Appl. Opt. 36, 2401-2407 (1997).

13. P. Jia, J. Kofman, and C. English, "Two-step triangularpattern phase-shifting method for three-dimensional objectshape measurement," Opt. Eng. 46, 083201 (2007).

14. P. Jia, J. Kofman, and C. English, "Multiple-step triangularpattern phase-shifting and the influence of number of steps and pitch on measurement accuracy," Appl. Opt. 46, 3253-3262 (2007).

15. P. S. Huang and S. Zhang, "Fast three-step phase-shifting algorithm," Appl. Opt. 45, 5086-5091 (2006).

16. S. Zhang and P. S. Huang, "Phase error compensation for a $3 \mathrm{D}$ shape measurement system based on the phaseshifting method," Opt. Eng. 46, 063601 (2007).

17. S. Zhang and S. Yau, "Generic nonsinusoidal phase error correction for three-dimensional shape measurement using a digital video projector," Appl. Opt. 46, 36-43 (2007).

18. K. Wu, J. Xi, Y. Yu, and Z. Yang, " $3 \mathrm{D}$ profile measurement based on estimation of spatial shifts between intensity ratios from multiple-step triangular patterns," Opt. Laser Eng. 51, $440-445$ (2013).

19. Z. Zhang, "A flexible new technique for camera calibration," IEEE Trans. Pattern Anal. Mach. Intell. 22, 1330-1334 (2000).

20. Y. Hu, J. Xi, E. Li, J. Chicharo, and Z. Yang, "Threedimensional profilometry based on shift estimation of projected fringe patterns," Appl. Opt. 45, 678-687 (2006).

21. S. Lei and S. Zhang, "Digital sinusoidal fringe generation: defocusing binary patterns vs focusing sinusoidal patterns," Opt. Laser Eng. 48, 561-569 (2010). 